\title{
p53-regulated long non-coding RNA PRECSIT promotes progression of cutaneous squamous cell carcinoma via STAT3 signaling
}

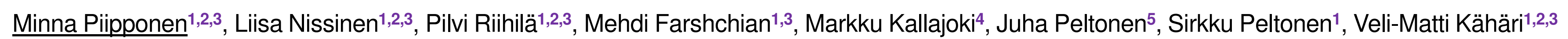
${ }^{1}$ Department of Dermatology and ${ }^{2}$ Cancer Research Laboratory, Western Cancer Centre of the Cancer Center Finland (FICAN West), University of Turku and Turku University Hospital, Turku, Finland; ${ }^{3}$ MediCity Research Laboratory, University of Turku, Turku, Finland; ${ }^{4}$ Department of Pathology, University of Turku and Turku University Hospital, Turku, Finland; ${ }^{5}$ Department of Cell Biology and Anatomy, University of Turku, Turku, Finland

\section{INTRODUCTION}

Long non-coding RNAs (IncRNAs) have emerged as functionally important mediators in a broad aspect of cellular regulation, from development and differentiation to emerging players in disease. They present a widely uncharacterized group of single-stranded RNA transcripts larger than 200 nucleotides in size, which interact with DNA, RNA or proteins, making them versatile regulators in all compartments of the cell. We have previously elucidated the mechanistic function of IncRNA PICSAR (p38 inhibited cutaneous squamous cell carcinoma associated lincRNA), which exerts a tumorigenic role in cutaneous squamous cell carcinoma (CSCC) $)^{1,2}$. However, the role of IncRNAs in CSCC in general is still largely unknown.

Here we have examined the role of IncRNA LINC00346, which is specifically overexpressed by CSCC cells in culture and in vivo. We show that LINC00346 is regulated by p53-signaling. We demonstrate that LINC00346 regulates the expression and activity of STAT3, which in turn upregulates the expression of matrix metalloproteinase (MMPs) MMP-1, MMP-3, MMP-10, and MMP-13 and promotes invasion of cSCC cells. Based on these observations LINC00346 was named p53 regulated carcinomaassociated STAT3 activating long intergenic non-protein coding transcript (PRECSIT)

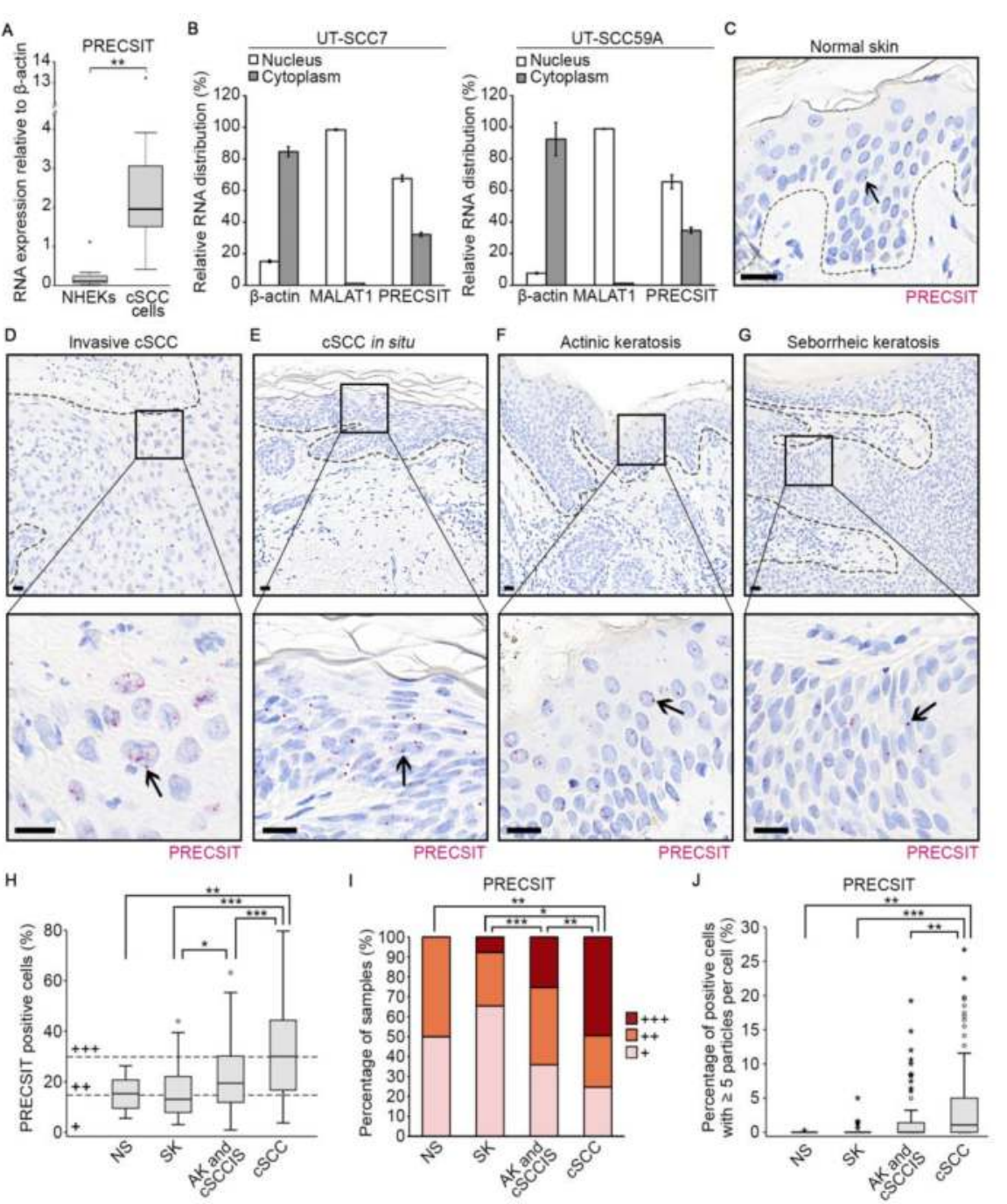

Figure 1. Expression of PRECSIT is specifically upregulated in cutaneous squamous cell carcinoma (cSCC) cells in culture and in vivo.

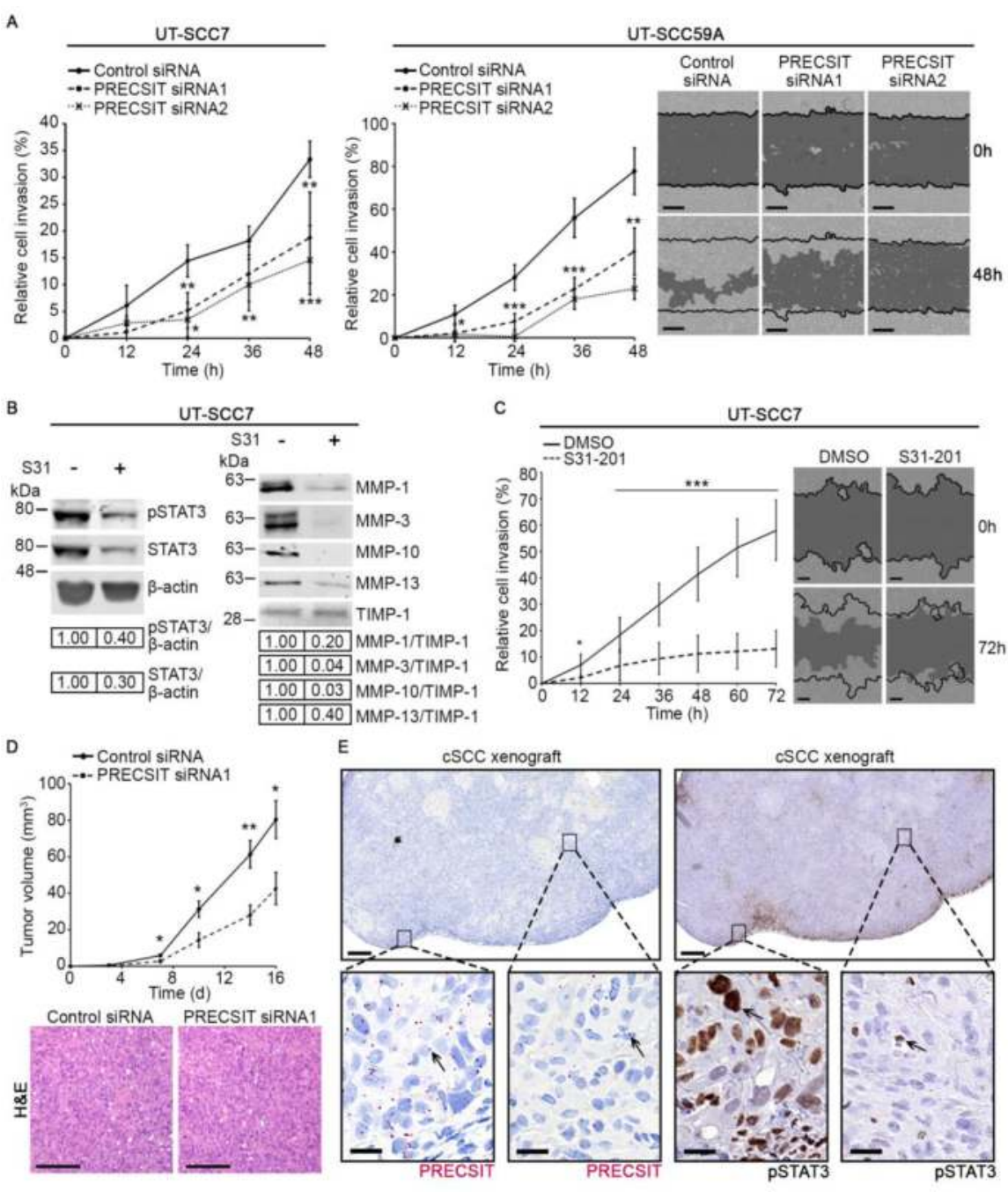

Figure 4. PRECSIT knockdown inhibits cSCC cell invasion and growth of CSCC xenografts in vivo.

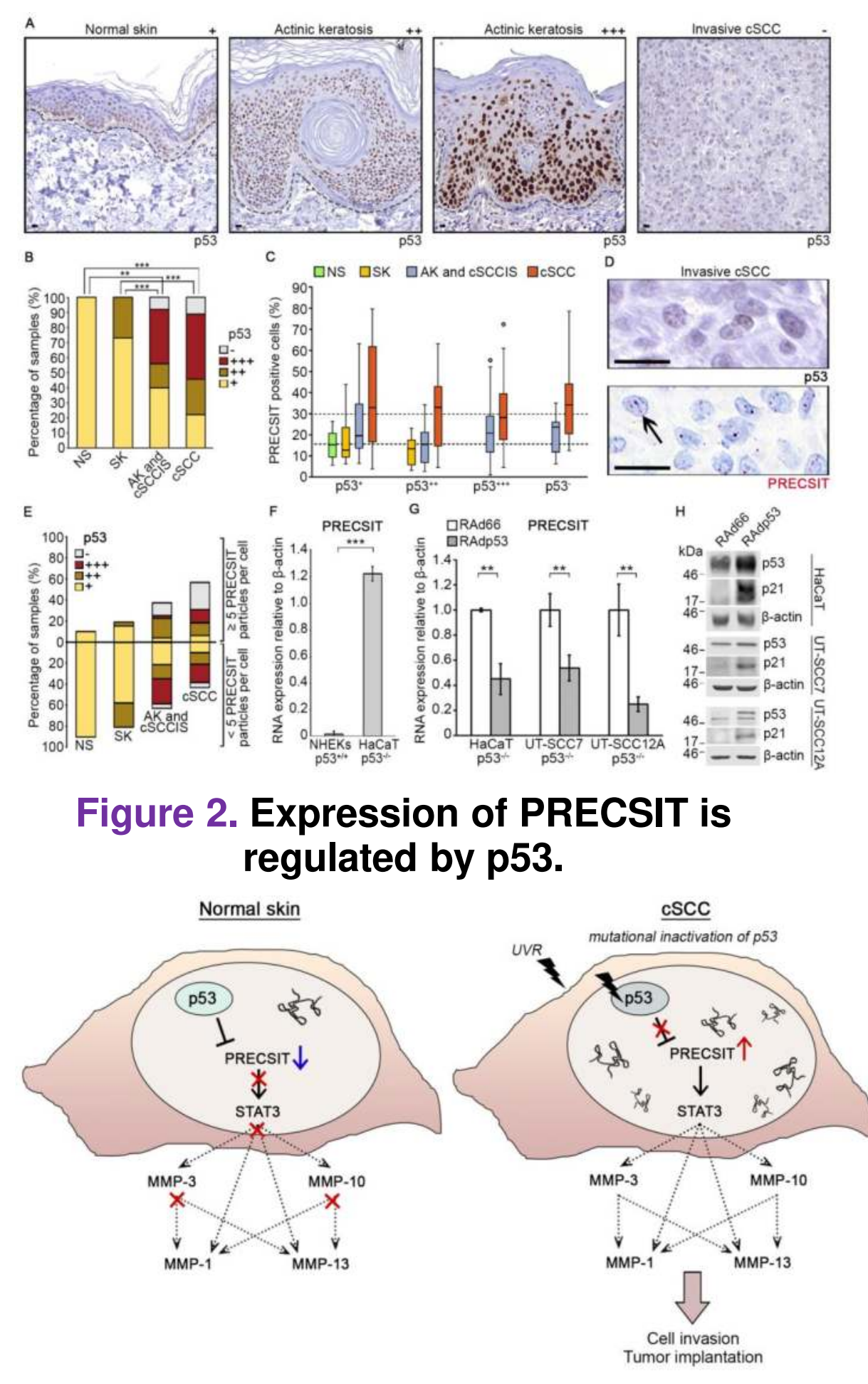

Figure 5. Proposed molecular model for the mechanistic role of PRECSIT in CSCC. Low level of PRECSIT expression is maintained in normal epidermal keratinocytes by functional p53. In cSCC cells mutational inactivation of p53, particularly leading to lack of functional p53 protein, results in upregulation and accumulation of PRECSIT in the nucleus. Elevated PRECSIT expression contributes to STAT3 activation and upregulation of the matrix metalloproteinases MMP-1, MMP-3, MMP-10 and MMP-13. MMP-3 and MMP-10 are capable of degrading several extracellular matrix (ECM) components, including basement membrane type IV collagen, fibronectin and laminin. They also activate latent collagenases MMP-1 and MMP-13, capable of cleaving fibrillar collagens type I and III in the dermal ECM. The proteolytic remodeling of ECM and basement membrane by MMPs is essential for cSCC cell invasion and tumor cell implantation, and it may also promote progression of premalignant and noninvasive malignant lesions AK and cSCCIS to fully invasive and metastatic CSCC.

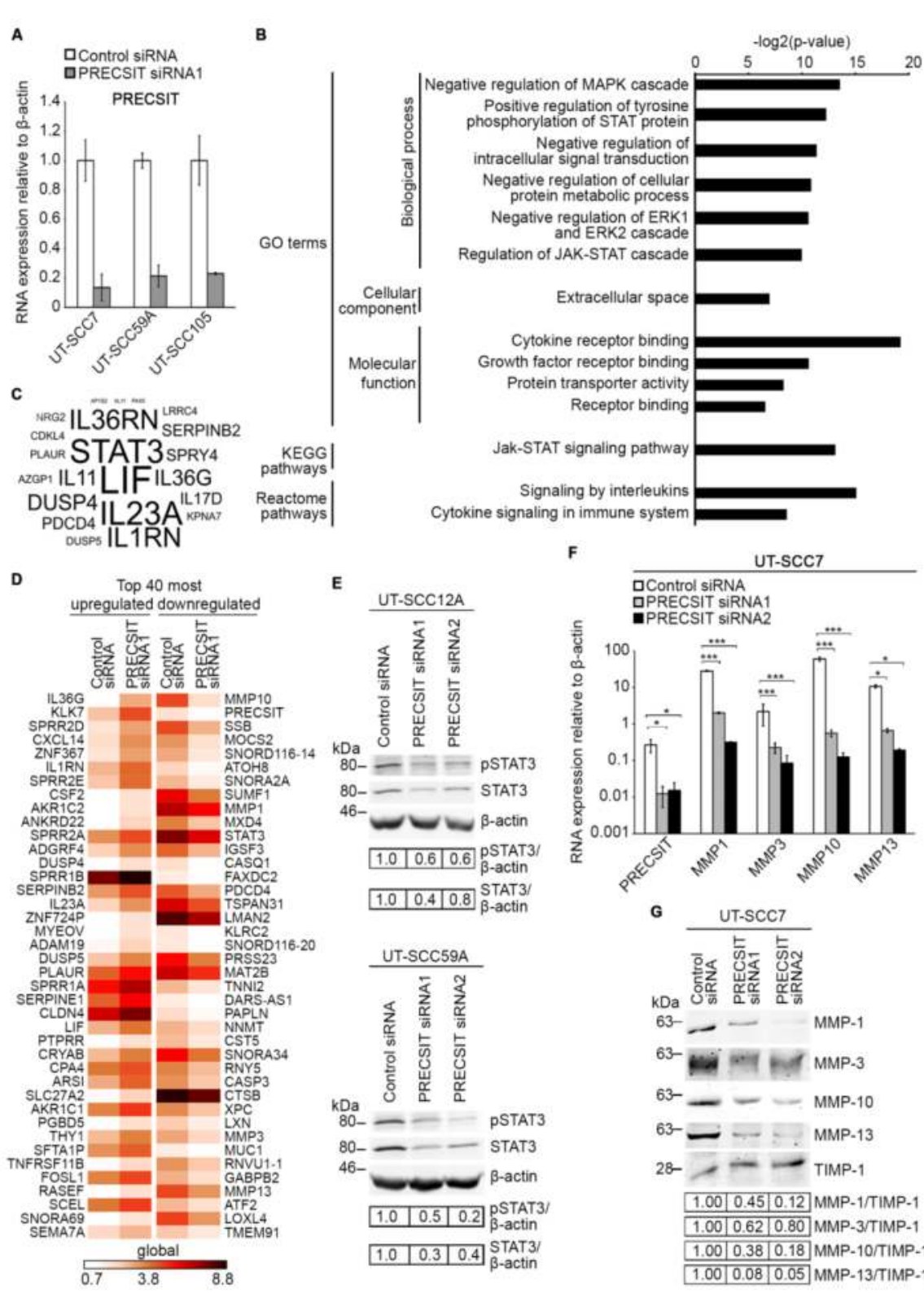

Figure 3. PRECSIT regulates expression of activated STAT3 and matrix metalloproteinases.

\section{CONCLUSION}

- PRECSIT is a p53-regulated, nuclear-enriched long non-coding RNA with induced expression in cSCC cells compared to NHEKs

- Expression of PRECSIT is induced in cSCC and precursor lesions compared to normal skin in vivo

- The level of activated transcription factor STAT3 is upregulated by PRECSIT and this in turn upregulates the expression of several invasion-associated MMPs

- PRECSIT promotes progression of cSCC by specifically regulating invasion of cSCC cells via STAT3 signaling 